\title{
Decentralized connectivity maintenance in mobile networks with bounded inputs
}

\author{
Dimos V. Dimarogonas and Karl H. Johansson
}

\begin{abstract}
A distributed control law that guarantees connectivity maintenance in a network of multiple mobile agents is presented. The control law respects the limited sensing capabilities of the agents by allowing each agent to take into account only agents within its sensing radius in the controller implementation. In contrast to previous approaches to the problem, the proposed control law does not attain infinite values whenever an edge tends to be lost, and is therefore a bounded distributed control law. This is achieved via the use of decentralized navigation functions which are potential fields with guaranteed boundedness and are redefined in this paper in order to take into account the connectivity maintenance objective. We first treat the case of connectivity maintenance in a static initial position based communication graph and extend the results to the case of dynamic edge addition. The results are then applied to a formation control problem.
\end{abstract}

\section{INTRODUCTION}

Recent work on multi-agent cooperative control has paid much attention to the consensus and formation control problems. The consensus problem involves convergence of all agents to a common value in the state space in a distributed fashion. Agents in this case can represent for example nodes in a large scale communication network [9],[6]. In the formation control case agents usually represent multiple robots or vehicles that aim to converge to a specified pattern in the state space. The desired formation can be either static [1],[8] or moving with constant velocity [18],[19].

A common assumption in distributed cooperative control problems is the connectedness of the underlying network, i.e. that there exists a path connecting any pair of nodes. While this seems a valid assumption in the case of communication networks, it is not realistic in the case of mobile sensor/vehicle networks, where communication between a pair of nodes is usually distance dependent. Motivated by this, several recent papers [9], [10],[5],[21],[4],[2] considered the distributed connectivity maintenance problem. A common approach of these papers is the use of unbounded potential fields that force agents that constitute a distance based communication link, to remain within this distance for all time. In particular, allowing the potential force between such pairs of agents to grow unbounded whenever agents tend to move away from the communication threshold distance

The authors are with KTH ACCESS Linnaeus Center, School of Electrical Engineering, Royal Institute of Technology (KTH),Stockholm, Sweden dimos, kallej@ee.kth.se. This work was done within TAISAURES program (297316-LB704859), funded by the Swedish Governmental Agency for Innovation Systems (VINNOVA) and Swedish Defence Materiel Administration (FMV). It was also supported by the Swedish Research Council, the Swedish Foundation for Strategic Research, and the EU NoE HYCON. provided a guarantee for edge maintenance and thus, connectivity maintenance. In practical situations however, the use of unbounded actuation is impossible. The use of potential fields with guaranteed boundedness is hence a desirable property in almost every application.

Motivated by this observation, this paper presents a control strategy that handles the distributed connectivity maintenance problem using bounded inputs. Specifically, we use a (by definition) bounded potential field that resembles the Navigation Function potential fields introduced in [12] and later extended to a centralized [14],[13],[11] and a decentralized [3],[20] multi-robot framework. The proposed bounded control law (i) maintains the edges that are formed based on the agents' initial positions and (ii) drives the agents to a common point in the state space. An application of the proposed framework to a formation stabilization problem is also presented. While the results involve only single integrator agents, we argue that the proposed development can also be applied to the nonholonomic and double integrator cases. These however are left for future publication endeavors.

The rest of the paper is organized as follows: in Section II the problem treated in this paper is presented. Section III begins with the matrix analysis tools used in the paper and process to present the Decentralized Navigation Function (DNF) based control law which is responsible for the bounded input connectivity maintenance objective, as well as the corresponding stability analysis. The case of dynamic edge addition is treated in Section IV while the proposed framework is applied to a formation control problem in Section V. Computer simulations are included in Section VI and the results of the paper are summarized in Section VII.

\section{Problem Formulation}

Consider $N$ single integrator point agents in the plane, described by kinematics of the form

$$
\dot{q}_{i}=u_{i}, i \in \mathcal{N}=[1, \ldots, N]
$$

where $q_{i} \in \mathbb{R}^{2}$ denotes the position and $u_{i} \in \mathbb{R}^{2}$ denotes the velocity (control input) for each agent $i \in \mathcal{N}$.

Each agent has limited sensing capabilities encoded by a cyclic sensing radius $d$ around it which is assumed common for all agents. Hence each agent is aware only of the positions of agents within its sensing radius. In order to encode the sensing limitations, graph theoretic notions are used. We thus assume that each agent is assigned with a specific subset $N_{i}$ of the rest of the team, called agent $i$ 's communication set, that includes the agents with which it can communicate in 
order to achieve the desired objective. Inter-agent communication is encoded in terms of a communication graph [17]:

Definition 1: The communication graph $\mathcal{G}=\{V, E\}$ is an undirected graph consisting of a set of vertices $V=$ $\{1, \ldots, N\}$ indexed by the team members and a set of edges, $E=\left\{(i, j) \in V \times V \mid i \in N_{j}\right\}$ containing pairs of nodes that represent inter-agent communication specifications.

The control objective is the construction of bounded control laws that drive the agents to consensus while maintaining the connectivity properties induced by the inter-agent relative initial conditions. The same problem was treated in [9],[10] for single integrator and in [5] for nonholonomic agents, however, the control law in these papers obtained unbounded values whenever pairs of agents that formed an edge tended to leave the sensing zone of one another. Real mobile agents however have limited actuator capabilities and hence boundedness of the control inputs is an issue not to be neglected. We propose here a framework based on the Navigation Function approach of [12], further explored in [11],[13], [3]. The control law of [12] is by default bounded, and thus respects the required actuator limitations.

\section{Control Design For Bounded Connectivity PRESERVING}

\section{A. Elements from Matrix Analysis}

We now review tools from matrix analysis [15] and graph theory [7] that we use in the sequel.

For a graph $\mathcal{G}$ with $n$ vertices the adjacency matrix $A=$ $A(\mathcal{G})=\left(a_{i j}\right)$ is the $n \times n$ matrix given by $a_{i j}=1$, if $(i, j) \in$ $E$ and $a_{i j}=0$, otherwise. If there is an edge connecting two vertices $i, j$, i.e. $(i, j) \in E$, then $i, j$ are called adjacent. When there is an orientation defined on each edge $(i, j) \in E$, the graph is called directed otherwise it is called undirected. A path of length $r$ from a vertex $i$ to a vertex $j$ is a sequence of $r+1$ distinct vertices starting with $i$ and ending with $j$ such that consecutive vertices are adjacent and that respects the orientation of the edges in the case of a directed graph. If there is a path between any two vertices of the graph $\mathcal{G}$, then $\mathcal{G}$ is called strongly connected in the case of directed, and simply connected in the case of undirected graphs. A directed graph has a spanning tree if there exists at least one vertex to which there exists a path from all other vertices respecting the edge orientation. The graph $\mathcal{G}=(V, E)$ corresponding to a real $n \times n$ matrix $M$ is a graph with $n$ vertices indexed by $1, \ldots, n$ such that there is an edge between vertices $i, j \in V$ if and only if $M_{i j} \neq 0$, i.e. $(i, j) \in E \Leftrightarrow M_{i j} \neq 0$.

A $n \times n$ real matrix with non-positive off-diagonal elements and zero row sums is called a Metzler matrix. It is shown in [15] that all the eigenvalues of a symmetric Metzler matrix are non-negative and zero is a trivial eigenvalue. The multiplicity of zero as an eigenvalue of a symmetric Metzler matrix is one (i.e. it is a simple eigenvalue) if and only if the corresponding undirected graph is connected. The trivial corresponding eigenvector is the vector of ones, $\overrightarrow{\mathbf{1}}$. The extension of these results to not necessarily symmetric Metzler matrices was provided in [16]. In this paper, we use the following Corollary of Theorem 1 in [16]:
Corollary 1: Assume that the $n \times n$ time-varying matrix $A(t)$ is Metzler for all $t \geq 0$ and that its elements are piecewise continuous and bounded with respect to time. Further assume that the time varying graph corresponding to $A(t)$ is strongly connected for all $t \geq 0$. Then the system $\dot{x}=-A(t) x$ converges to an agreement equilibrium, i.e. an equilibrium where all the elements of the vector $x$ are equal.

In fact, the Corollary holds in the weaker case where $A(t)$ contains a spanning tree sufficiently often. However, we use the strong connectedness assumption since this is the one that is only needed for the validity of the main results of this paper. Moreover, the condition of Corollary 1 is equivalent to the fact that 0 is a simple eigenvalue of $A(t)$ for all $t \geq 0$, with corresponding eigenvector the vector of ones, $\overrightarrow{\mathbf{1}}$.

\section{B. Control Design and Convergence Analysis}

Each agent is equipped with a decentralized navigation function-like potential field of the form $\varphi_{i}: \mathbb{R}^{2 N} \rightarrow[0,1]$

$$
\varphi_{i}=\frac{\gamma_{i}}{\left(\gamma_{i}^{k}+G_{i}\right)^{1 / k}}
$$

where $k>0$ is a positive scalar and $\gamma_{i}, G_{i}$ are discussed in the sequel. Note that $\varphi_{i}$ is bounded, taking values in $[0,1]$.

The term $\gamma_{i}$ is the term to be minimized for the fulfillment of the agreement objective. For this reason each agent's communication set $N_{i}$ is defined as the set of agents that are initially located within the sensing zone of agent $i$ :

$$
N_{i}=\left\{j \in \mathcal{N}, j \neq i:\left\|q_{i}(0)-q_{j}(0)\right\|<d\right\} .
$$

This definition of $N_{i}$ justifies the fact that the communication graph, as defined in Definition 1, is undirected, since (3) implies that $i \in N_{j} \Leftrightarrow j \in N_{i}$ for all $i, j \in \mathcal{N}$. By showing that for all pairs of agents $(i, j)$ s.t. $\left\|q_{i}(0)-q_{j}(0)\right\|<d$ the proposed controller guarantees that $\left\|q_{i}(t)-q_{j}(t)\right\|<d$ for all $t>0$, the edges are guaranteed to remain invariant (i.e. agents $i, j$ remain within distance $d$ from one another) and hence the communication graph itself, remains invariant throughout the closed loop system evolution. This result is shown explicitly in the sequel.

Based on the fact that all agents initially located within distance $d$ from each other remain within this distance for all time, the set $N_{i}$ is a static set. Hence no new edges are created even when an agent not initially located within the sensing radius of another, enters inside this set. The case of dynamic graphs will be discussed in the next section.

Hence, $N_{i}$ is static in this section. Function $\gamma_{i}$ is defined:

$$
\gamma_{i} \triangleq \sum_{j \in N_{i}} \frac{1}{2}\left\|q_{i}-q_{j}\right\|^{2}
$$

and is minimized whenever the agreement objective with respect to agent $i$ has been fulfilled. The function $G_{i}$ is responsible for edge maintenance and is defined as

$$
G_{i} \triangleq \prod_{j \in N_{i}} \beta_{i j} \triangleq \prod_{j \in N_{i}} \frac{1}{2}\left(d^{2}-\left\|q_{i}-q_{j}\right\|^{2}\right)
$$


The control law of each agent $i$ is now defined by

$$
u_{i}=-K_{i} \frac{\partial \varphi_{i}}{\partial q_{i}}
$$

where $K_{i}>0$ is a positive gain.

Using the notation $\nabla_{i}(\cdot) \triangleq \frac{\partial}{\partial q_{i}}(\cdot)$, we can compute

$$
\begin{aligned}
& \frac{\partial \varphi_{i}}{\partial q_{i}}=\frac{\left(\gamma_{i}^{k}+G_{i}\right)^{1 / k} \nabla_{i} \gamma_{i}-\frac{\gamma_{i}}{k}\left(\gamma_{i}^{k}+G_{i}\right)^{1 / k-1}\left(k \gamma_{i}^{k-1} \nabla_{i} \gamma_{i}+\nabla_{i} G_{i}\right)}{\left(\gamma_{i}^{k}+G_{i}\right)^{2 / k}} \\
& \Rightarrow \frac{\partial \varphi_{i}}{\partial q_{i}}=\left(\gamma_{i}^{k}+G_{i}\right)^{-1 / k-1}\left(G_{i} \nabla_{i} \gamma_{i}-\frac{\gamma_{i}}{k} \nabla_{i} G_{i}\right)
\end{aligned}
$$

Note that since $\gamma_{i}$ and $G_{i}$ never attain the zero value simultaneously, the control law does not become infinite whenever two agents forming an edge tend to the distance $d$ from one another. Thus, unlike previous results on closed loop connectivity maintenance, the control design of this paper allows for boundedness of the control laws each time a link between two agents tends to be broken. This is of course due to the fact that the potential field $\varphi_{i}$ itself, does not attain infinite values whenever $G_{i} \rightarrow 0$, for any $i \in \mathcal{N}$.

The first result states that the control law forces agents that are initially located within distance $d$ from each other to remain within this distance for all time. Hence the definition of $N_{i}$ is rendered meaningful since each agent $i$ does not have to violate its sensing constraints in order to sense agents within $N_{i}$. The result is stated as follows:

Lemma 2: The set $G_{i}>0, \forall i \in \mathcal{N}$, is invariant for the trajectories of (1) under the control law (4).

Proof: At a point $q_{0}$ on the set $G_{i}=0$, we have

$$
\frac{\partial \varphi_{i}}{\partial q_{i}}\left(q_{0}\right)=\left(\gamma_{i}^{k}\right)^{-1 / k-1}\left(-\frac{\gamma_{i}}{k} \nabla_{i} G_{i}\right)
$$

The partial derivative of $G_{i}$ with respect to $q_{i}$ is

$$
\nabla_{i} G_{i}=\sum_{j \in N_{i}} \bar{\beta}_{i j} \nabla_{i} \beta_{i j}=-\sum_{j \in N_{i}} \bar{\beta}_{i j}\left(q_{i}-q_{j}\right)
$$

where $\bar{\beta}_{i j} \triangleq \prod_{\substack{l \in N_{i} \\ l \neq j}} \beta_{i l}$. Since $G_{i}\left(q_{0}\right)=0$, then $\beta_{i j}\left(q_{0}\right)=0$ for at least one $j \in N_{i}$. If there exists $j \in N_{i}$ for which $\beta_{i j}\left(q_{0}\right)=0$ and $\left.\beta_{i k}\left(q_{0}\right)\right) \neq 0$, for all $k \in N_{i}, k \neq j$, then $\bar{\beta}_{i j}>0$. Hence, in this case $\frac{\partial \varphi_{i}}{\partial q_{i}}$ is nonsingular at $q_{0}$. Since $\varphi_{i}$ is also a smooth function, the result in this case follows from the Implicit Function Theorem. The negated gradient motion $-\frac{\partial \varphi_{i}}{\partial q_{i}}$ is normal to the surface $G_{i}=0$ and hence points towards the set $G_{i}>0$. When there exists at least two agents $k, j \in N_{i}, k \neq j$ for which $\beta_{i j}\left(q_{0}\right)=\beta_{i k}\left(q_{0}\right)=0$, then $\frac{\partial \varphi_{i}}{\partial q_{i}}\left(q_{0}\right)=0$. But $\varphi_{i}: \mathbb{R}^{2 N} \rightarrow[0,1]$ and $\varphi_{i}\left(q_{0}\right)=$ $\frac{\gamma_{i}\left(q_{0}\right)}{\left(\gamma_{i}^{k}\left(q_{0}\right)+G_{i}\left(q_{0}\right)\right)^{1 / k}}=1$ which means that at $q_{0}, \varphi_{i}$ achieves its maximum. However, since the set of initial conditions $G_{i}>0$ is open, and no open set of initial conditions can be attracted to the maxima of $\varphi_{i}$ along the negative gradient motion $-\frac{\partial \varphi_{i}}{\partial q_{i}}$ [11], we conclude that the set $G_{i}>0$ is invariant for all $i \in \mathcal{N} . \diamond$

Hence, if agent $i$ starts in the set $G_{i}>0$ it remains within it for all time. Since $G_{i}$ tends to zero whenever an initially formed edge is bound to break, i.e., whenever an agent $j \in N_{i}$ tends to leave the sensing radius of $i$, the invariance of $G_{i}>0$ implies that all agents initially located within distance $d$ from $i$, remain within this distance for all time. Since this takes place simultaneously for all agents all edges are maintained and the connectivity of the initially formed communication graph remains invariant. We also note that in the navigation function framework of [12],[13],[11],[3],[20] the set $G_{i}$ was defined as the collision free set for agent $i$. Lemma 2 is thus similar to the corresponding ones in the aforementioned papers. The difference is that the definition of $G_{i}$ in the current paper establishes edge maintenance.

Next, we show that (4) leads to agreement in the case of an initially connected communication graph. The partial derivative with respect to $q_{i}$ of $\gamma_{i}$ is given by $\nabla_{i} \gamma_{i}=$ $\sum_{j \in N_{i}}\left(q_{i}-q_{j}\right)$. From the proof of Lemma 2, we have $\nabla_{i} G_{i}=$ $-\sum_{j \in N_{i}} \bar{\beta}_{i j}\left(q_{i}-q_{j}\right)$, where $\bar{\beta}_{i j} \triangleq \prod_{\substack{l \in N_{i} \\ l \neq j}} \beta_{i l}$. We then have

$$
\begin{aligned}
& G_{i} \nabla_{i} \gamma_{i}-\frac{\gamma_{i}}{k} \nabla_{i} G_{i}= \\
& =G_{i} \sum_{j \in N_{i}}\left(q_{i}-q_{j}\right)+\frac{\gamma_{i}}{k} \sum_{j \in N_{i}} \bar{\beta}_{i j}\left(q_{i}-q_{j}\right)= \\
& =\sum_{j \in N_{i}} \bar{\beta}_{i j}\left(\beta_{i j}+\frac{\gamma_{i}}{k}\right)\left(q_{i}-q_{j}\right)= \\
& =\sum_{j \in N_{i}} \pi_{i j}\left(q_{i}-q_{j}\right)
\end{aligned}
$$

where $\pi_{i j} \triangleq \bar{\beta}_{i j}\left(\beta_{i j}+\frac{\gamma_{i}}{k}\right)$. Note that while in general $\pi_{i j} \neq$ $\pi_{j i}$, Lemma 2 guarantees that $\pi_{i j}>0$ and $\pi_{j i}>0$, for all $j \in N_{i}$. For each agent we now have

$$
\begin{aligned}
& \dot{q}_{i}=-K_{i}\left(\gamma_{i}^{k}+G_{i}\right)^{-1 / k-1} \sum_{j \in N_{i}} \pi_{i j}\left(q_{i}-q_{j}\right)= \\
& =-\sum_{j \in N_{i}} \mu_{i} \pi_{i j}\left(q_{i}-q_{j}\right)
\end{aligned}
$$

with $\mu_{i} \triangleq K_{i}\left(\gamma_{i}^{k}+G_{i}\right)^{-1 / k-1}>0$. The closed-loop system can now be written in stack vector form as

$$
\dot{q}=-\left(P(q) \otimes I_{2}\right) q
$$

where the matrix $P(q)$ is defined as

$$
P_{i j}=\left\{\begin{array}{l}
\sum_{j \in N_{i}} \mu_{i} \pi_{i j}, i=j \\
-\mu_{i} \pi_{i j}, j \in N_{i} \\
0, j \notin N_{i}
\end{array}\right.
$$

The matrix $P$ has zero row sums. Since its off diagonal elements are non-positive, $P$ is a Metzler matrix with zero row sums. The stability properties of (5) have been extensively studied in [16] and since $P_{i j}<0$ implies $P_{j i}<0$ for all $j \in N_{i}$, the directed graph corresponding to $P$ is strongly connected if and only if the initially formed undirected communication graph is connected, and that happens due to the fact that an initially formed edge between $i$ and $j$ is both invariant and bidirectional. In essence, if the agents start from the set $G_{i}>0, \forall i \in \mathcal{N}$, the matrix $P$ trivially satisfies the conditions of Corollary 1 (and of Theorem 1 in [16]) provided that the initially formed communication graph is connected. Hence if the graph is initially connected, the agents reach a common value in the state space. The above conclusion is summarized in the following Theorem: 
Theorem 3: Assume that (1) is driven by (4) and that the initially formed communication graph under ruling (3) is connected. Further assume that $G_{i}(0)>0$ for all $i \in \mathcal{N}$. Then the agents reach a common point in the state space.

\section{DynAmic Edge AdDition}

The previous section involved the case where the communication graph considered was static, i.e., no new edges were added whenever an agent, not initially located within the sensing zone of another, entered its sensing zone. In practical situations however, it is more convenient to consider creation of new edges whenever an agent enters the sensing zone of another. This leads to a faster convergence rate and corresponds to a more realistic formulation of the problem. On the other hand, allowing each agent to consider every agent as a neighbor once this agent enters its neighboring set leads to an increase of computational load. Hence there is a tradeoff between faster convergence rate and computational effort. We encode the tradeoff discussed previously by allowing each agent to have a maximum number of neighbors $M$. The main result of this section shows that in fact each agent will have exactly $M$ neighbors at steady state.

In this section, the communication set of agent $i$ initially includes agents that belong to its sensing zone at $t=0$ :

$$
N_{i}(0)=\left\{j \in \mathcal{N}, j \neq i:\left\|q_{i}(0)-q_{j}(0)\right\|<d\right\}
$$

In order to add new edges, we assume that a new edge is created each time a new agent enters a subset of the sensing zone of $i$. In particular, we define the set:

$$
N_{i}^{*}(t)=\left\{\begin{aligned}
j & \in \mathcal{N}, j \neq i: \\
\left(j \notin N_{i}\left(t^{-}\right)\right) & \wedge\left(\left\|q_{i}(t)-q_{j}(t)\right\|<d-\varepsilon\right)
\end{aligned}\right\}
$$

where $\varepsilon>0$ a small positive scalar. Then, $N_{i}^{*}(t) \subseteq N_{i}(t)$. We also make the following assumption:

Assumption 1: The parameter $M$ is chosen so that there exists a realization of the graph with $N$ vertices where all the vertices have exactly $M$ adjacent vertices.

This assumption is a viability condition on the edge addition ruling that will now be defined. Specifically, the communication set of each agent $i$ is updated as follows:

$$
N_{i}(t)=\left\{\begin{array}{l}
N_{i}\left(t^{-}\right) \cup N_{i}^{*}(t), \text { if }\left|N_{i}\left(t^{-}\right)\right|<M \\
N_{i}\left(t^{-}\right), \text {otherwise }
\end{array}\right.
$$

The function $G_{i}$ is now defined as:

$$
G_{i}=\prod_{j \in N_{i}(t)} \frac{1}{2}\left(d^{2}-\left\|q_{i}-q_{j}\right\|^{2}\right)
$$

and the control law of each agent is now defined as:

$$
u_{i}=-\sum_{j \in N_{i}(t)} \mu_{i} \pi_{i j}\left(q_{i}-q_{j}\right)
$$

and is updated when a new agent enters $N_{i}$.

Since when a new edge is created the function $G_{i}$ remains strictly positive, the edge maintenance result of Lemma 2 still holds and hence whenever two agents form a new link, i.e. are at a distance less than $d-\varepsilon$ for the first time, they remain within distance $d$. Thus, the definition of dynamic edge addition respects the limited sensing capabilities of all agents. Thus, once a new edge is created it is never lost. We are now ready to state the main result of this section:

Theorem 4: Assume that (1) is driven by (8) and that the initially formed communication graph under ruling (6) is connected. Also assume that $N_{i}(0) \leq M$ for all $i \in \mathcal{N}$ and that Assumption 1 holds. Then the agents reach a common point in the state space. Moreover, $\lim _{t \rightarrow \infty}\left|N_{i}(t)\right|=M, \forall i \in$ $\mathcal{N}$, i.e., all agents have exactly $M$ neighbors at steady state. Proof: If that no new edges are added, then Theorem 3 holds, i.e. all agents converge to the same point in the workspace. However, this means that all agents eventually come to a distance $d-\varepsilon$ from one another. It is then evident that all agents attain the maximum number of neighbors, $M$, allowed by the edge addition ruling (7), while converging to a common point in the state space. $\diamond$

The above framework guarantees that connectedness is maintained and at the same time allows each agent to add new neighbors until the number of neighbors of each agent is exactly $M$. Moreover, the number of final neighbors $M$ can be different for each agent $i$, with a slight modification of the rule (7) and Assumption 1. Finally, the control law (8) satisfies the actuator boundedness requirement.

\section{Connectivity Preserving Formation Control}

In this section we apply the results of section III to a formation stabilization problem. Each agent $i$ 's objective is to converge to a particular relative position vector $c_{i j}$ with respect to each $j \in N_{i}$. Thus, a vector $c_{i j} \in \mathbb{R}^{2}$ is associated to each edge $(i, j) \in E$ of the communication graph $\mathcal{G}=(V, E)$, and this specifies the agent relative positions in the desired final formation. The desired formation is called feasible if (i) it belongs to the set $\Phi \triangleq$ $\left\{q \in W \mid q_{i}-q_{j}=c_{i j}, \forall(i, j) \in E\right\}$ of all possible desired formation configurations and (ii) $\Phi$ is nonempty.

According to (3), only pairs of agents $i, j$ that are initially within distance $d$ are allowed to be assigned a desired relative formation vector $c_{i j}$. In order not to violate this, we require that $\left\|c_{i j}\right\|<d$, for all $(i, j) \in E$. The formation control law for each agent $i$ is given by

$$
u_{i}=-K_{i} \frac{\partial \varphi_{i}^{f}}{\partial q_{i}}
$$

where

$$
\varphi_{i}^{f}=\frac{\gamma_{i}^{f}}{\left(\left(\gamma_{i}^{f}\right)^{k}+G_{i}^{f}\right)^{1 / k}}
$$

is the connectivity preserving formation decentralized navigation function. The functions $\gamma_{i}^{f}$ and $G_{i}^{f}$ are now redefined in order to treat the formation control objective as follows:

$$
\gamma_{i}^{f} \triangleq \sum_{j \in N_{i}}\left(\left\|q_{i}-q_{j}-c_{i j}\right\|\right)^{2}
$$

and

$$
G_{i}^{f} \triangleq \prod_{j \in N_{i}} \beta_{i j}^{f} \triangleq \prod_{j \in N_{i}} \frac{1}{2}\left(\left(d-\left\|c_{i j}\right\|\right)^{2}-\left\|q_{i}-q_{j}-c_{i j}\right\|^{2}\right)
$$


Similarly to the analysis of the static graph case, the formation control law can be calculated as

$$
u_{i}=-\sum_{j \in N_{i}} \mu_{i}^{f} \pi_{i j}^{f}\left(q_{i}-q_{j}-c_{i j}\right)
$$

where $\mu_{i}^{f} \triangleq K_{i}\left(\left(\gamma_{i}^{f}\right)^{k}+G_{i}^{f}\right)^{-1 / k-1}$ and $\pi_{i j}^{f} \triangleq$ $\bar{\beta}_{i j}^{f}\left(\beta_{i j}^{f}+\frac{\gamma_{i}^{f}}{k}\right)$, with $\bar{\beta}_{i j}^{f} \triangleq \prod_{\substack{l \in N_{i} \\ l \neq j}} \beta_{i l}^{f}$. Lemma 2 has the following counterpart in the formation control case:

Lemma 5: The set $G_{i}^{f}>0, \forall i \in \mathcal{N}$, is invariant for the trajectories of the system (1) under the control law (9).

Proof: The proof is exactly the same as that of Lemma 2 if one replaces $\varphi_{i}$ with $\varphi_{i}^{f}, \gamma_{i}$ with $\gamma_{i}^{f}$ and $G_{i}$ with $G_{i}^{f}$. $\diamond$

Thus, $G_{i}^{f}>0$ is invariant for all $i \in \mathcal{N}$. Hence, for all $(i, j) \in E$ we have $\left(d-\left\|c_{i j}\right\|\right)^{2}-\left\|q_{i}-q_{j}-c_{i j}\right\|^{2}>$ 0 , and since $\left\|c_{i j}\right\|<d$, the last inequality doesn't violate the connectivity rule $\left\|q_{i}-q_{j}\right\|<d$, since $\left(d-\left\|c_{i j}\right\|\right)^{2}-$ $\left\|q_{i}-q_{j}-c_{i j}\right\|^{2}>0 \stackrel{\left\|c_{i j}\right\|<d}{\Rightarrow}\left\|q_{i}-q_{j}-c_{i j}\right\|<d-\left\|c_{i j}\right\| \Rightarrow$ $\left|\left\|q_{i}-q_{j}\right\|-\left\|c_{i j}\right\|\right|<d-\left\|c_{i j}\right\| \Rightarrow\left\|q_{i}-q_{j}\right\|<d$.

Assuming that the desired formation is feasible, we can choose a random global coordinate frame and denote by $c_{i}$ the configuration of agent $i$ in a desired formation configuration with respect to this global coordinate frame, for all $i \in \mathcal{N}$. Then $c_{i j}=c_{i}-c_{j}, \forall(i, j) \in E$ for all possible desired formations. Define $q_{i}-q_{j}-c_{i j}=q_{i}-q_{j}-\left(c_{i}-c_{j}\right)=\tilde{q}_{i}-\tilde{q}_{j}$, i.e. $\tilde{q}_{i}=q_{i}-c_{i}$ for all $i \in \mathcal{N}$. Using the new coordinates, $G_{i}^{f}, \gamma_{i}^{f}$ can be written as functions of the $\tilde{q}$ variables. Moreover, since $\dot{\tilde{q}}_{i}=\dot{q}_{i}-\dot{c}_{i}=\dot{q}_{i}=u_{i}=-\sum_{j \in N_{i}} \mu_{i}^{f} \pi_{i j}^{f}\left(\tilde{q}_{i}-\tilde{q}_{j}\right)$, the closed loop system in the $\tilde{q}$ space is given by

$$
\dot{\tilde{q}}=-\left(P^{f}(\tilde{q}) \otimes I_{2}\right) \tilde{q}
$$

where $\tilde{q}=\left[\tilde{q}_{1}^{T}, \ldots, \tilde{q}_{N}^{T}\right]^{T}$, and the matrix $P^{f}$ is defined in the exact similar way as matrix $P$ as follows

$$
P_{i j}^{f}=\left\{\begin{array}{l}
\sum_{j \in N_{i}} \mu_{i}^{f} \pi_{i j}^{f}, i=j \\
-\mu_{i}^{f} \pi_{i j}^{f}, j \in N_{i} \\
0, j \notin N_{i}
\end{array}\right.
$$

In essence, the closed loop system (12) behaves in the $\tilde{q}$ space as (5) behaves in the $q$ space, i.e., Theorem 3 holds in the $\tilde{q}$ space for system (12). Thus, (12) reaches a configuration where the elements of the stack vector $\tilde{q}$ are equal, i.e. $\tilde{q}_{i}=$ $\tilde{q}_{j}=\tilde{q}^{*}$ for all $i, j \in \mathcal{N}$. Thus, $q_{i}-q_{j}=\tilde{q}_{i}-\tilde{q}_{j}+c_{i}-c_{j}=$ $\tilde{q}^{*}-\tilde{q}^{*}+c_{i j} \Rightarrow q_{i}-q_{j}=c_{i j}, \forall(i, j) \in E$. We conclude that the agents converge to the desired formation. The above observations are summarized in the following Theorem:

Theorem 6: Assume that the multi-agent system is driven by the control law (9) and that the initially formed communication graph under ruling (3) is connected. Further assume that $\left\|c_{i j}\right\|<d$, for all $(i, j) \in E$ and that the desired formation is feasible. Then the initially formed edges are maintained, i.e., pairs of agents initially within a distance $d$ from one another remain within this distance for all time, and the multi-agent team reaches the desired formation.

\section{Simulations}

The results are now supported with computer simulations.

In the first simulation, six agents evolve under the control (4) and the rule (3). The initially formed graph is connected and remains invariant by virtue of Lemma 2. In Figure 1, the agents' initial positions are marked with a cross. In this example, $d=0.36$. Note that although agents 1 and 2 are initially very close to losing their link due to the existence of the two subgroups on their left and right side respectively, the controller forces them to maintain it. The network stays connected and agents converge to an agreement point in accordance with Theorem 3. The boundedness of the inputs

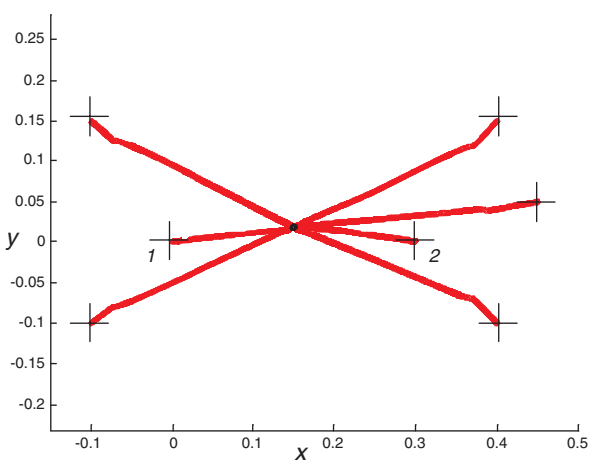

Fig. 1. Connectivity preserving state agreement with bounded inputs control law (4) and initial condition rule (3).

of agents 1 and 2 that tend to break their link is depicted in the velocity diagrams of Figure 2. The first two graphs show the plots of the coefficients of the velocities (control inputs) of agents 1 and 2 in the $x, y$ directions while the bottom plot shows the evolution of their velocity norms.

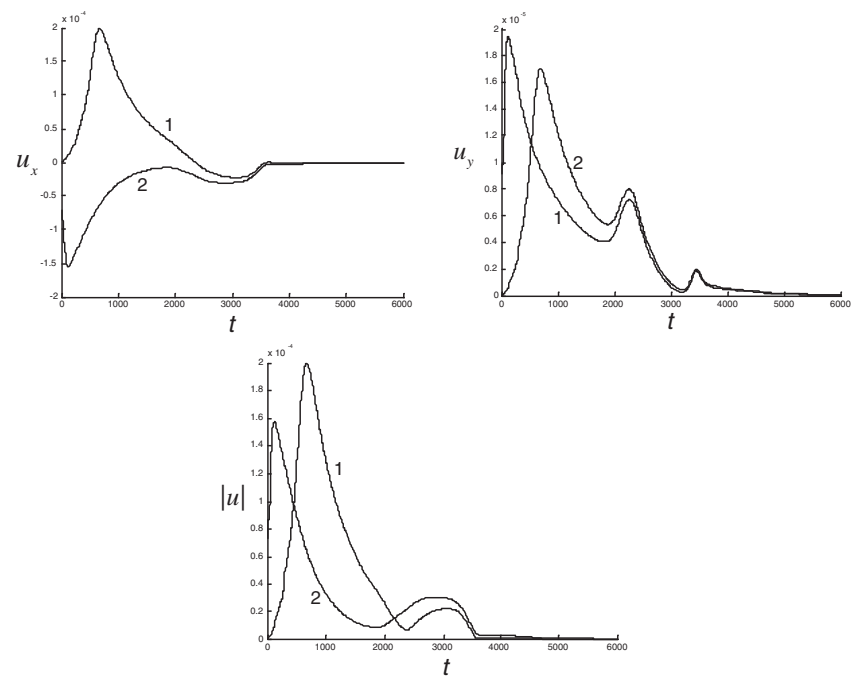

Fig. 2. Velocity Diagrams. The first two graphs show the plots of the coefficients of the velocities (control inputs) of agents 1 and 2 in the $x, y$ directions while the bottom plot shows the evolution of the velocity norms of the agents 1 and 2 in time.

The next simulation (Figure 3) is a formation control 
example. Seven agents navigate under the control (11). The communication graph formed based on (3) is a line graph. The sensing radius is given by $d=0.0025$ while formation objective is convergence to a straight line, where each agent will be at a distance equal to $\left\|c_{i j}\right\|=0.002<d$ from each of its neighbors. In Figure 3, the crosses represent the initial positions of the agents and their final locations are noted by a black circle. Moreover the trajectory of each agent is the line that connects its initial and final configuration. As witnessed in the figure, the agents eventually reach the desired line formation. Figure 4 verifies that the interagent distances do in fact converge to the desired distance $\left\|c_{i j}\right\|=0.002<d$. The figure shows the plot of the inter-agent distances of three pairs of agents that form an edge in the initially formed communication graph. All distances converge to 0.002 .

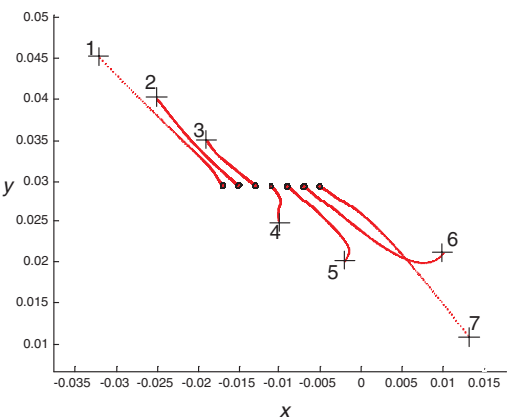

Fig. 3. Connectivity preserving formation control under the initial condition ruling (3) and the control law (11).

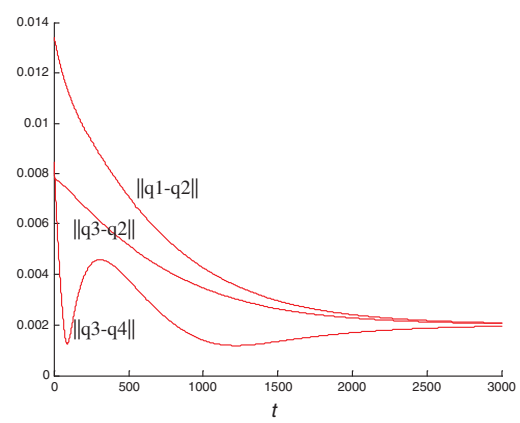

Fig. 4. Inter-agent distances of three pairs of agents that form an edge in the initially formed communication graph. All distances converge to the expected desired value of 0.002 .

\section{CONCLUSIONS}

A distributed control law that guarantees connectivity maintenance in a network of multiple mobile agents was presented. The control law respects the limited sensing capabilities of the agents by allowing each agent to take into account only agents within its sensing radius in the controller implementation. In contrast to previous approaches on the problem, the proposed control law does not attain infinite values whenever an edge tends to be lost, and is therefore a bounded distributed control law. This is achieved via the use of decentralized navigation functions which are potential fields with guaranteed boundedness and are modified in this paper in order to treat the connectivity maintenance objective. We first treated the case of connectivity maintenance in a static initial position based communication graph and extended the results to the case of dynamic edge addition. The results were then applied to a formation control problem.

\section{REFERENCES}

[1] B.E. Bishop. Dynamics-based control of robotic swarms. IEEE Intern. Conf. Robotics and Automation, pages 2763-2768, 2006.

[2] M.C. DeGennaro and A. Jadbabaie. Decentralized control of connectivity for multi-agent systems. 45th IEEE Conf. Decision and Control, pages 3628-3633, 2006.

[3] D. V. Dimarogonas, S. G. Loizou, K.J. Kyriakopoulos, and M. M. Zavlanos. A feedback stabilization and collision avoidance scheme for multiple independent non-point agents. Automatica, 42(2):229243, 2006.

[4] D.V. Dimarogonas and K.J. Kyriakopoulos. Connectivity preserving distributed swarm aggregation for multiple kinematic agents. 46th IEEE Conf. Decision and Control, 2007. 2913-2918.

[5] D.V. Dimarogonas and K.J. Kyriakopoulos. On the rendezvous problem for multiple nonholonomic agents. IEEE Transactions on Automatic Control, 52(5):916-922, 2007.

[6] L. Fang, P.J. Antsaklis, and A. Tzimas. Asynchronous consensus protocols: Preliminary results,simulations and open questions. 44th IEEE Conf. Decision and Control, pages 2194-2199, 2005.

[7] C. Godsil and G. Royle. Algebraic Graph Theory. Springer Graduate Texts in Mathematics \# 207, 2001.

[8] M.A. Hsieh and V. Kumar. Pattern generation with multiple robots. IEEE Intern. Conf. Robotics and Automation, pages 2442-2447, 2006.

[9] M. Ji and M. Egerstedt. Connectedness preserving distibuted coordination control over dynamic graphs. 2005 American Control Conference, pages 93-98.

[10] M. Ji and M. Egerstedt. Distributed coordination control of multiagent systems while preserving connectedness. IEEE Transactions on Robotics, 23(4):693-703, 2007.

[11] C.S. Karagoz, H.I. Bozma, and D.E. Koditschek. Coordinated navigation of multiple independent disk-shaped robots. Tech. report no. ms-cis-07-16, Department of Computer and Information Science, University of Pennsylvania, 2007.

[12] D. E. Koditschek and E. Rimon. Robot navigation functions on manifolds with boundary. Advances Appl. Math., 11:412-442, 1990.

[13] S. G. Loizou and K. J. Kyriakopoulos. Closed loop navigation for multiple holonomic vehicles. Proc. of IEEE/RSJ Int. Conf. on Intelligent Robots and Systems, pages 2861-2866, 2002.

[14] S.G. Loizou and K.J Kyriakopoulos. Navigation of multiple kinematically constrained robots. IEEE Transactions on Robotics, 2008. to appear.

[15] D.G. Luenberger. Introduction to Dynamic Systems: Theory, Models and Applications. John Wiley \& Sons, 1979.

[16] L. Moreau. Stability of continuous-time distributed consensus algorithms. 43rd IEEE Conf. Decision and Control, pages 3998-4003, 2004.

[17] A. Muhammad and M. Egerstedt. Connectivity graphs as models of local interactions. Journal of Applied Mathematics and Computation, 168(1):243-269, 2005

[18] R. Olfati-Saber. Flocking for multi-agent dynamic systems: Algorithms and theory. IEEE Transactions on Automatic Control, 51(3):401-420, 2006.

[19] H.G. Tanner, A. Jadbabaie, and G.J. Pappas. Flocking in fixed and switching networks. IEEE Transactions on Automatic Control, 52(5):863-868, 2007.

[20] H.G. Tanner and A. Kumar. Formation stabilization of multiple agents using decentralized navigation functions. Robotics: Science and Systems, 2005.

[21] M.M. Zavlanos and G.J. Pappas. Distributed connectivity control of mobile networks. 46th IEEE Conf. Decision and Control, pages 35913596, 2007. 